\title{
Extend aortic repair for acute type A aortic dissection with rupture and malperfusion complicated with Ehlers-Danlos syndrome
}

\author{
Koki Maekawa ${ }^{1}$, Toshiki Fujiyoshi ${ }^{1}$, Masaki Kano ${ }^{1}$, Ryumon Matsumoto ${ }^{1}$, Yu Nakano ${ }^{1}$, \\ and Hitoshi Ogino ${ }^{1}$ \\ ${ }^{1}$ Tokyo Medical University
}

July 14, 2021

\begin{abstract}
Background Ehlers-Danlos syndrome (EDS) is a disorder in the metabolism of fibrillary collagen. Its vascular type (vEDS) frequently develops arterial rupture and aortic dissection. Case presentation The patient was a 54-year-old gentleman who suffered from cardiac tamponade, malperfusion of the left carotid artery and the right lower extremity due to acute type A aortic dissection. Rupture of the aortic root and a huge entry located from the transverse arch to the proximal descending aorta were found. There were no significant deformities at aortic valve, so we tried extended repairs of valve sparing root replacement (VSRR) and total arch replacement (TAR) with frozen elephant trunk (FET) and were successfully performed. He was finally diagnosed as vascular Ehlers-Danlos syndrome (vEDS) by genetic examination. Conclusion Emergency extensive repairs of VSRR and TAR with FET for critical AAAD with rupture and malperfusion was successfully performed for a vEDS patient.
\end{abstract}

\section{Extend aortic repair for acute type $\mathbf{A}$ aortic dissection with rupture and malperfusion} complicated with Ehlers-Danlos syndrome

\section{Extend aortic repair}

Koki Maekawa, MD, Toshiki Fujiyoshi, MD, PhD, Masaki Kano, MD, Yu Nakano, MD, Ryumon Matsumoto, MD, Hitoshi Ogino, MD, PhD

Department of Cardiovascular Surgery, Tokyo Medical University, Tokyo, Japan

Conflict of Interests: All authors declare that they have no conflict of interests.

Meeting Presentation: None

Funding statement: The authors declare no conflicts of interest associated with this manuscript.

Institutional review board approval or waiver: Waivered because this is case presentation.

Patient consent statement: Written informed consent was obtained from the patient for publication of this case report and accompanying images.

Corresponding author:

Hitoshi Ogino, MD, PhD Cardiovascular Surgery, Tokyo Medical University

6-7-1 Nishishinjuku, Shinjukuku, Tokyo, 160-0023 JAPAN

E-mail: hogino@tokyo-med.ac.jp

Phone: (+81) 3-3342-6111 


\section{Abstract \\ Background}

Ehlers-Danlos syndrome (EDS) is a disorder in the metabolism of fibrillary collagen. Its vascular type (vEDS) frequently develops arterial rupture and aortic dissection.

\section{Case presentation}

The patient was a 54-year-old gentleman who suffered from cardiac tamponade, malperfusion of the left carotid artery and the right lower extremity due to acute type A aortic dissection. Rupture of the aortic root and a huge entry located from the transverse arch to the proximal descending aorta were found. There were no significant deformities at aortic valve, so we tried extended repairs of valve sparing root replacement (VSRR) and total arch replacement (TAR) with frozen elephant trunk (FET) and were successfully performed. He was finally diagnosed as vascular Ehlers-Danlos syndrome (vEDS) by genetic examination.

\section{Conclusion}

Emergency extensive repairs of VSRR and TAR with FET for critical AAAD with rupture and malperfusion was successfully performed for a vEDS patient.

\section{Keywords}

vascular Ehlers-Danlos syndrome, acute aortic dissection, emergency surgery

\section{Introduction}

Ehlers-Danlos syndrome (EDS) is a disorder in the metabolism of fibrillary collagen. Its vascular type (vEDS) frequently develops arterial rupture and aortic dissection [1]. In general, a tear-oriented surgery has been recommended for acute type A aortic dissection (AAAD).

However, with increased experiences of total arch replacement (TAR), its prevalence has recently increased, particularly, in conjunction with frozen elephant trunk (FET) [2]. In the proximal site, in cases with rupture or dilatation of the root or with primary entry in the root, concomitant aortic root repairs are required. Of them, valve sparing root replacement (VSRR) is much more challenging due to prolonged cardiac arrest and cardiopulmonary bypass (CPB). We report on successful extended repairs of VSRR and TAR with FET for massive AAAD with root rupture and multiple malperfusion in a difficult patient with vEDS.

\section{Case Report}

The patient was a 54-year-old gentleman. On arrival, his conditions were critical with blood pressure of $67 / 47 \mathrm{mmHg}$. Echo examinations showed pericardial effusion and dissecting flaps in the bilateral carotid arteries. Computed tomographic (CT) scans showed AAAD with malperfusion of the left carotid artery and the right common iliac artery. At surgery, CPB was immediately established with a right femoro-femoral circuit. A large amount of clot was found in the intrapericardial space. Central cannulation for CPB into the true channel of the ascending aorta was added. At 28 of the bladder temperature, hypothermic circulatory arrest (HCA) was induced, and the heart was arrested. A huge primary entry was located from the transverse arch to the proximal descending aorta at the zone 4 level (Fig 1 ). The 
dissection was extended into each arch-vessel. The proximal descending aorta was transected completely at the zone 4 level, excluding the primary entry. A FET device, FROZENIX ${ }^{\circledR}$ (Japan Lifeline Co, Tokyo, Japan) of $23 \mathrm{~mm}$ in diameter and $60 \mathrm{~mm}$ in length, was inserted into the true lumen. To this aortic stump, a multibranched arch graft, Gelweave $^{\text {TM }}$ (TERUMO VASCUTEK, Glasgow, UK) of $24 \mathrm{~mm}$ in diameter, was connected. After systemic reperfusion, the three arch-vessels were reconstructed, repairing all dissected arteries. At the proximal site, rupture of the aortic root was found at the commissure between the right- and the non-Valsalva sinuses (Fig $2 \mathbf{A}$ ). The dissection was extended into the right coronary artery. Aortic valve had no significant deformities, which allowed us to perform VSRR using a reimplantation technique with Gelweave Valsalva ${ }^{\mathrm{TM}}$ Ante-Flo (TERUMO VASCUTEK, Glasgow, UK) of $26 \mathrm{~mm}$ in diameter. The commissure post between the right and non-coronary cusps had no adventitial. Only the dissecting flap was carefully fixed to the graft with a 5-0 polypropylene pledgetted mattress suture. The $2^{\text {nd }}$-low sutures for attachment of the aortic wall were also meticulously placed (Fig 2B ). He developed delay of full awakening. The CT-scans and MRI revealed small cerebral infarction on the left frontal lobe and the cerebellum, however it was not so critical without any permanent neurological dysfunctions. The postoperative CT-scans (Fig 3 ) showed no remarkably abnormal findings. He was finally diagnosed as vEDS by a genetic test, revealing a novel frame-shift mutation of the COL3A1 gene.

\section{DISCUSSION}

vEDS is a hereditary syndrome caused by impaired metabolism of fibrous collagen. Because

of its vascular fragility, surgical outcomes are generally poor [3]. VSRR was challenging

because it is more technically demanding [4]. Particularly for vEDS, there are only a few reports about emergency case with AAAD [5]. VSRR was however attempted, because of relatively young age and no significant deformities of the aortic valve, and of the surgeon's (HO) experiences over 200 VSRRs. No adventitia was preserved at the commissure between the right- and non-coronary cusps. Careful attachment of such a destroyed and fragile commissure to the graft was required. Apart from remodeling procedures, the commissure posts are inserted in the graft and attached to its inside, which is one of the advantages of reimplantation techniques. During surgery, it was suspected that the patient had a sort of connective tissue disease because of his tissue fragility. Finally, it was revealed as vEDS. This is the first report on a vEDS patient having VSRR and TAR with FET for AAAD successfully performed. In vEDS, severe follow-up of the anastomosis is necessary because of the fragility of the tissue.

\section{References}

1. Pepin M, Schwarze U, Superti-Furga A, et al. Clinical and genetic feature of Ehlers-Danlos syndrome type IV, the vascular type. N Engl J Med. 2000;342:673-80.

2. Uchida N, Shibamura H, Katayama A, Shimada N, Sutoh M. Total arch replacement with an open stent graft for acute type A aortic dissection: fate of the false lumen. Eur J Cardiothorac Surg. 2009;35:83-8.

3. Brooke BS, Arnaoutakis G, McDonnell NB, Black JH $3^{\text {rd }}$. Contemporary management of vascular complications associated with Ehlers-Danlos syndrome. J Vasc Surg 2010;51:131-8. 
4. Bethea BT, Fitton TP, Cameron DE, et al. Results of aortic valve-sparing operations: experience with remodeling and reimplantation procedures in 65 patients. Ann Thorac Surg. 2004;78(3):767-72.

5. Papadopoulos G, Maier O, Bonner F, Veulemans V. Vascular type of Ehlers-Danlos syndrome: a case report of an aortic dissection during pregnancy. Am J Case Rep. 2019;20:233-7.

\section{Figure legends}

\section{Fig 1.}

A large entry was located from the arch to the proximal descending aorta (a dotted line). The small arrow shows orifice of the left subclavian artery.

\section{Fig 2 A.}

The aortic root was massively dissected with ruptured adventitia (big arrow) around the commissure (small arrow) between the right and non-coronary Valsalva sinuses. The dissection was extended into the right coronary artery (triangle).

\section{Fig 2B.}

Good coaptation of the aortic valve was confirmed with appropriate attachment of the ruptured commissure (arrow) to the graft.

\section{Fig 3.}

Postoperative CT-scan shows VSRR and TAR using FET with closure of the false lumen in the proximal descending aorta (arrow). 


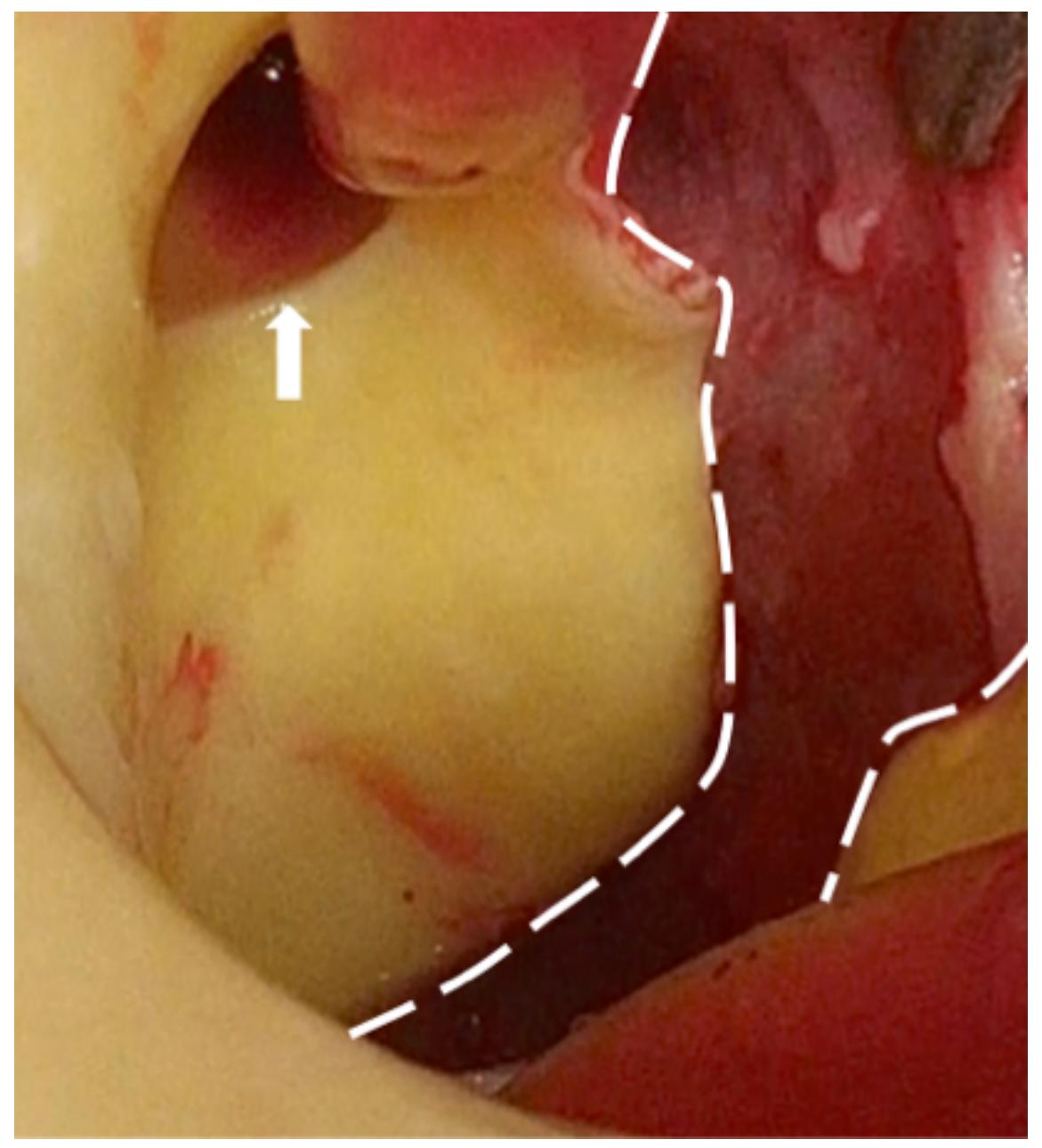



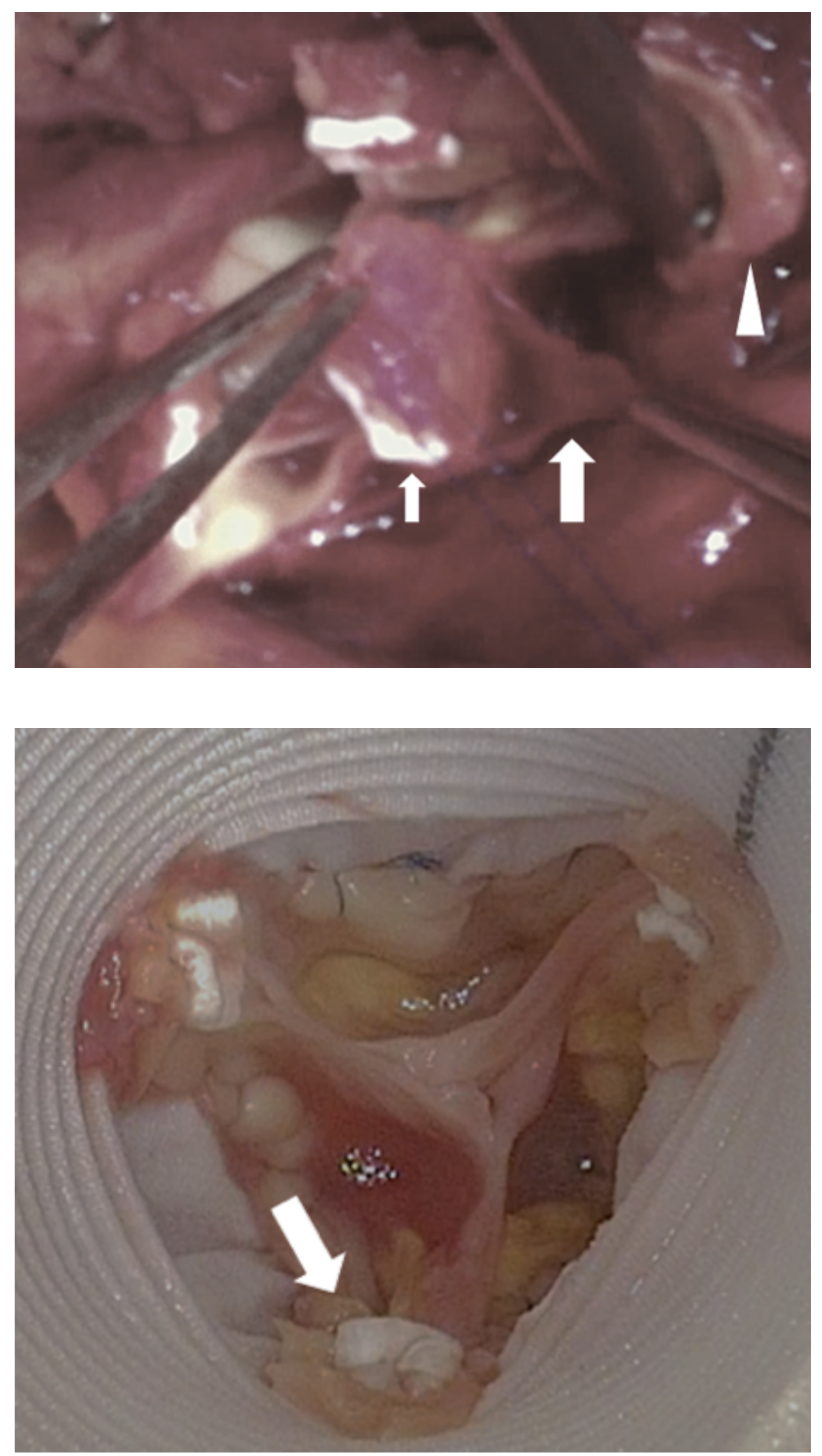


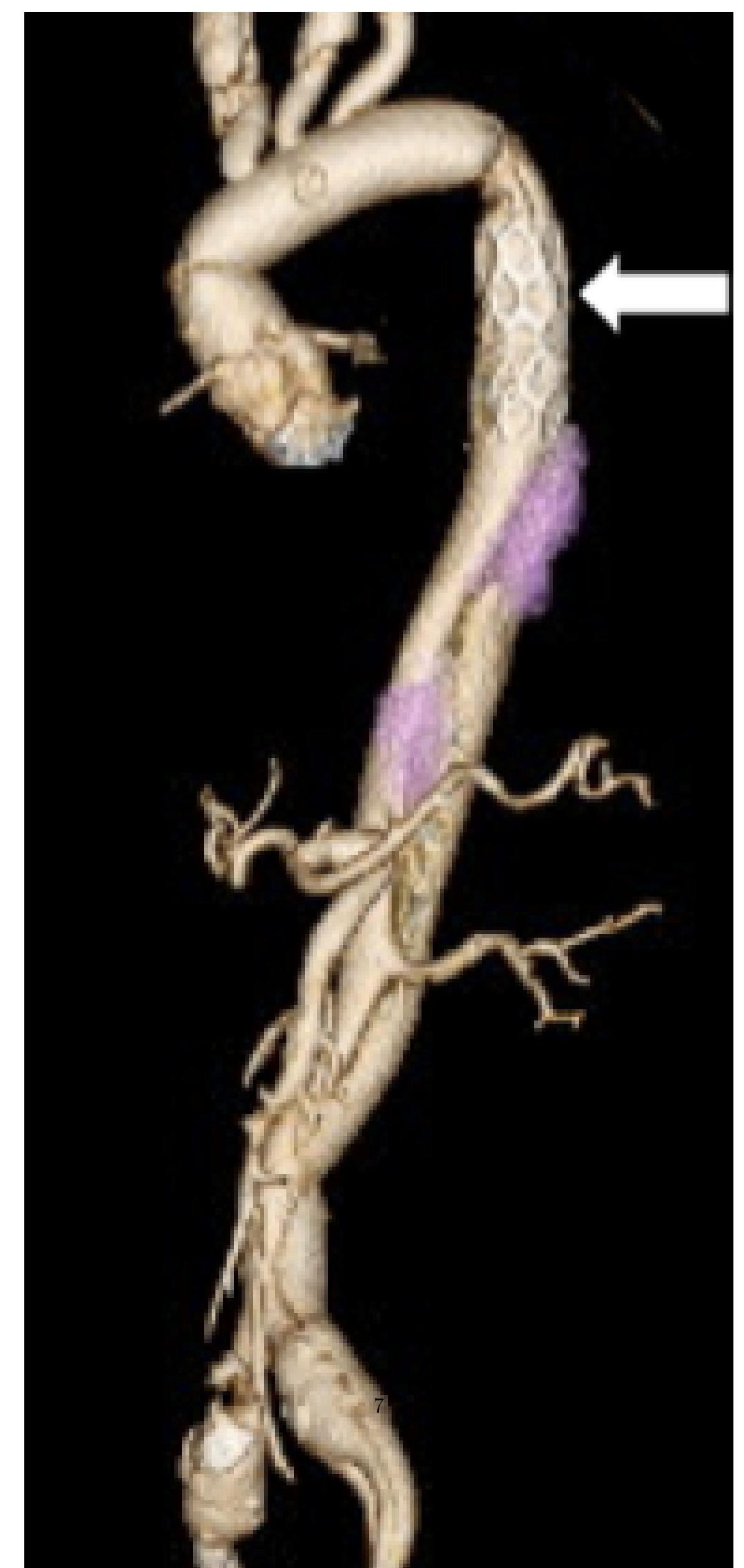

